\title{
Commissioning and Calibration of a Photoemission Electron Microscope
}

Falk Niefind and Sujitra Pookpanratana

Nanoscale Device and Characterization Division, National Institute of Standards and Technology, Gaithersburg, Maryland, United States

Since its inception in the 1930s, photoemission electron microscopy (PEEM) has emerged as a powerful addition to the surface scientist's toolbox. 1 The general operating procedure is as follows: the sample is illuminated by a light source of sufficient photon energy to drive the photoelectric effect, thus generating photoemitted electrons. These electrons are subsequently guided by the electron optics system onto a detector, where the final image is produced.Currently, a new commercial PEEM is entering service at NIST, allowing us to go beyond classical real space imaging. It incorporates a magnetic prism array that allows the user to fully resolve the energy and momenta (E-k) of the photoelectrons thus the ability of $\mu \mathrm{m}$ scale angle-resolved photoemission (ARPES).2 A PEEM image usually displays contrast deriving from both topographic and electronic contrast. For graphene on silicon carbide samples, this contrast manifests in different regions of intensity. We assigned these to the buffer layer, 1-2 layer graphene and graphite. As the PEEM measurements are non-destructive, different techniques such as Raman spectroscopy can be utilized to confirm the assignment after PEEM measurements. To quantify the contribution of graphene, buffer layer, and graphite on the sample surface, we implemented a simple, threshold-based image segmentation method. The thresholds used were determined by histogram intensity distribution, and the accuracy of segmenting the image will be discussed. We use a standard normal distribution approach to quantify the error that is inherent to the threshold-based segmentation method.We routinely achieve a spatial resolution of $50 \mathrm{~nm}$ with this PEEM without aberration corrections. However, the sample alignment and focusing of the PEEM is done manually by the researchers. Depending on the state of the instrument and the nature of the sample at hand, this procedure can take multiple hours of repetitive work and focusing results may vary with operator. We are currently implementing a Laplacian based algorithm to automate some of the tasks.Acknowledgements: We thank Dr. Randolph Elmquist for providing the epitaxial graphene for this study.

\section{References}

1. M. Dạbrowski, Y. Dai and H. Petek, Chem. Rev., 2020, 120, 6247-6287.

2. R. M. Tromp, Y. Fujikawa, J. B. Hannon, A. W. Ellis, A. Berghaus and O. Schaff, J. Phys. Condens. Matter, 2009, 21, 314007. 